\title{
Study of emergency care issues based on regions
}

\author{
Sicheng Zhu \\ School of North China Electric Power University, Hebei 071000, China \\ zsc4572@126.com
}

Keywords: Emergency; public satisfaction; Logistic.

\begin{abstract}
In this papers, we study the emergency problems. Under different circumstances, with 6 areas, for example, we discuss about how to allocate ambulances. We consider the emergency response time $\mathrm{T}$ as the deciding factor. When the accident occurred in any region, there will be one or more ambulances arriving at the accident sites within $\mathrm{T}$ time. For this reason, we introduce the concept of public satisfaction, which is a function of the independent variable time $t$ and a "S" curve in time. We choose logistic curve to describe public satisfaction. As time $t$ increase, public satisfaction decline at different rates, where the growth coefficient $r$ is proportional to the population in all regions. We consider the case that the accidents may occur in multiple areas, then we get the optimal solutions based on the public satisfaction.
\end{abstract}

\section{Introduction}

With the progress of urbanization, the urban regional emergency issues are increasingly prominent. Therefore, take 6 regions for example, to different regions, how to deploy a limited number of ambulances and an ambulance is parked in the right place so that more people in the 6 regions of the city can get emergency ambulance service in 8 minutes after calling the emergency number is an urgent issue.

Firstly, two important factors in planning emergency ambulances are traffic between different regions of the city and the population in each region. So the algorithm we use must take for these two important factors into account at the same time. For an indicator to evaluate a scheme, we refer to public satisfaction as judging scheme is good or bad. Public satisfaction is not only a function of time, and for different regions, regional populations will also affect the public satisfaction.

Secondly, when an emergency occurs, according to the implementation of the schemes, we can determine the optimal configuration of the emergency ambulances. Because different levels of accidents, the dispatch ambulances can be more than one. The standards we judge the feasibility of this scheme can be in accordance with the public satisfaction and the total time required for emergency ambulances to arrive at the accident site under the schemes.

\section{Models}

We assume that the time interval between two accidents is long and the two accidents have no effects and association with each other. Ignored the time for judging after receiving the emergency call. We need to reconfigure the scheme after an emergency occurs. We believe that the time required for dispatch is short, that is the dispatch process does not affect emergency care.

We set $A_{1}, A_{2}, \cdots, A_{n}$ for $n$ an emergency vehicle supply points. $B$ is the emergency locations, $X$ is the number of required emergency ambulances for $B$. During the emergency response time, $A_{i}$ can dispatch emergency ambulances $x_{i}$ and $x_{i}^{\prime}$ is the number of actual involvement in dispatching emergency ambulances, where $1 \leq i \leq n$ and $\sum_{i=1}^{n} x_{i}=X$. The time takes from $A_{i}$ to $B$ is $t_{i} . T$ is the emergency response time. $R_{j}$ is the population owned by the $j$ region, where $1 \leq j \leq m$ and $m$ is the total number of regions. The population we referred to is a fixed value. 
Public satisfaction (SAT) is a feedback parameters based on people's reflecting for the quality of the emergency ambulance service. Its feature should be like the "S" curve, that is during the emergency response time $T$ [1], SAT is essentially in a modest reduction and it is basically a fixed value. Beyond the scope of time $T$, SAT decreases significantly faster, that is public satisfaction shows a clear downward trend. As for the description for $S A T$, we select the classic logistic growth function curve [2]. Its differential equation is:

$$
\left\{\begin{array}{c}
\frac{d N}{d t}=-r\left(1-\frac{N}{K}\right) \mathrm{N} \\
N\left(\mathrm{t}_{0}\right)=\mathrm{N}_{0}
\end{array}\right.
$$

It solution is:

$$
N(\mathrm{t})=\frac{-K}{\frac{N_{0}-K}{N_{0}} e^{-r t_{0}+r t}+1}
$$

Where

$\mathrm{N}(\mathrm{t})$ stands for public satisfaction.

$r$ stands for the growth factor in different regions, which is decided by the number of population in the region. We believe $\mathrm{r}$ is proportional to the population of the region and we set $r=R_{j} / 10,000$.

$\mathrm{k}$ stands for upper limit of the curve $(\mathrm{K}=1)$.

$t_{0}$ stands for the emergency response time T, we set $N_{0}=0.8$ is the SAT at the moment $\mathrm{T}$.

There is curve of SAT about the function of the time in any region:

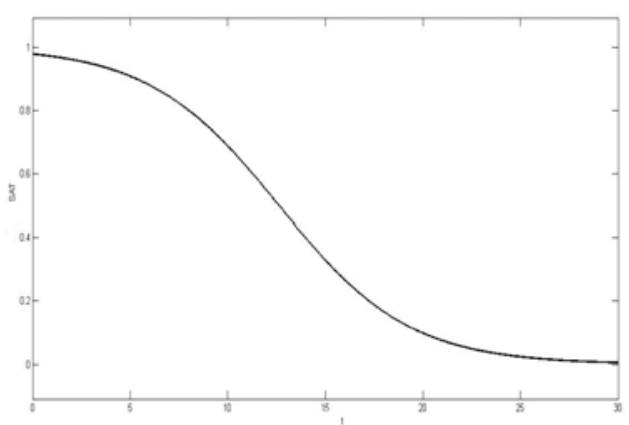

Fig1. The change trend of SAT

The emergency levels can be divided into four grades in turn $G_{1}, G_{2}, G_{3}, G_{4}$ [3]. The severity is $G_{4}>G_{3}>G_{2}>G_{1}$. We dispatch ambulances to rush to the accident sites based on the different emergency levels.

We set $S_{j}$ for the emergency operation scheme $j, t_{s i}\left(A_{1}, A_{2}, \cdots, A_{n}\right)$ is the time of each ambulances site to the emergency site in emergency operation scheme $j$. We judge the emergency level in view of the severity of the accidents. The $S A T_{j}$ is the public satisfaction for emergency operation scheme $j$, so the whole emergency operation schemes are $S=\left\{S_{1}, S_{2}, \cdots, S_{m}\right\}$.

\section{The result}

First, we assume that there are totally 3 ambulance supplies for these 6 regions' emergency care. Obviously, the most reasonable approach is that the three ambulances are available, that means no ambulance in dealing with emergencies. Also, we do not consider the schemes that two or three ambulances in the same region. On this premise, we analyze the possible 20 scenarios. The emergency level directly affect the number of ambulances required for the emergencies.

When the emergency level is $G_{1}$ or $G_{2}$, we need an ambulance for the accident site. And when the emergency levels are $G_{3}$ and $G_{4}$ respectively, we need two and three ambulances. For a certain 
scheme of ambulances distribution, we analyze the emergency response time in every one of the six regions and combine with regional population to obtain public satisfaction. After considering public satisfaction in the six regions, we can get the total public satisfaction of the scheme.

Integrated the public satisfaction of three conditions, we choose the best scheme, which is the placement of three ambulances when we cannot get to know the emergency levels.

We can get the public satisfaction $S A T$ curves about time of these 6 regions:

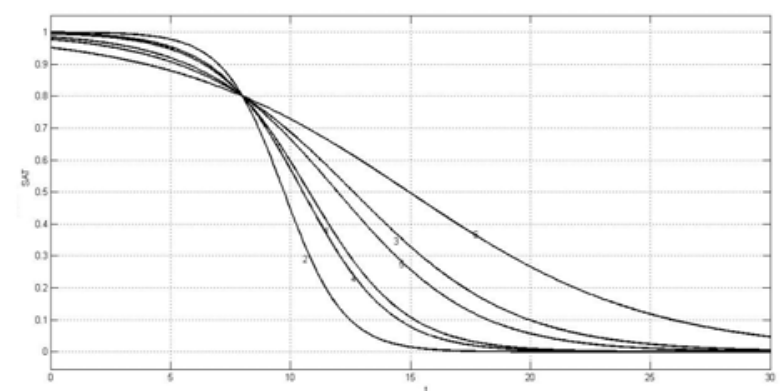

Fig2. The curves of SAT in these 6 regions

We take a region needed emergency care for example, we get the following result:

Tab 1: A region needs emergency care

\begin{tabular}{ccccccc}
\hline \multicolumn{2}{c}{ The emergency site number } & The total SAT & The total time & The average time & The time STD \\
\hline \hline 1 & 2 & 3 & 5.504 & 25.5 & 4.25 & 4.287 \\
1 & 2 & 4 & 5.225 & 29 & 4.833 & 4.956 \\
1 & 2 & 5 & 5.744 & 16 & 2.667 & 1.966 \\
1 & 2 & 6 & 5.129 & 22 & 3.667 & 4.227 \\
1 & 3 & 4 & 5.543 & 21.5 & 3.583 & 2.94 \\
1 & 3 & 5 & 5.632 & 18.5 & 3.083 & 2.616 \\
1 & 3 & 6 & 4.966 & 26.5 & 4.167 & 4.521 \\
1 & 4 & 5 & 5.618 & 18 & 3 & 2.683 \\
1 & 4 & 6 & 5.618 & 18 & 3 & 2.683 \\
1 & 5 & 6 & 5.596 & 21 & 3.5 & 2.51 \\
2 & 3 & 4 & 5.549 & 21.5 & 3.583 & 2.94 \\
2 & 3 & 5 & 5.638 & 18.5 & 3.083 & 2.616 \\
2 & 3 & 6 & 4.972 & 26.5 & 4.417 & 4.521 \\
2 & 4 & 5 & 5.624 & 18 & 3 & 2.683 \\
2 & 4 & 6 & 5.624 & 18 & 3 & 2.683 \\
2 & 5 & 6 & 5.602 & 21 & 3.5 & 2.51 \\
3 & 4 & 5 & 4.244 & 32.5 & 5.417 & 5.92 \\
3 & 4 & 6 & 4.244 & 32.5 & 5.417 & 5.92 \\
3 & 5 & 6 & 4.197 & 37.5 & 6.25 & 6.195 \\
4 & 5 & 6 & 3.925 & 39 & 6.5 & \\
\hline
\end{tabular}

According to the total public satisfaction when a region needs emergency care, the best configuration scheme for emergency ambulances is $\{1,2,5\}$.

Similarly, when two regions need emergency care, we can get the best schemes are $\{3,5,6\}$ and $\{4,5,6\}$, and when three regions need emergency care, the best scheme is $\{2,3,5\}$.

\section{Conclusion}

The evaluation criteria: according to the results, we not only get total public satisfaction, but also get total time and average time and the time STD. The average time can reflect the average case about 
the time for ambulances arriving to the emergency site. The time STD can reflect the balance of the time distribution required in all regions. We get the optimal schemes based on the total public satisfaction of three conditions, then we use the average time and the time STD to evaluate a certain scheme whether it is suit for other cases.

(1)According to the total satisfaction when a region needs emergency care, we can preliminary obtain the best ambulances configuration: $\{1,2,5\}$.

(1)When the scheme is used in case two, the total public satisfaction is 8.652, which is lower compared with other schemes. The time STD is 9.939, which is higher compared with other schemes. So the scheme $\{1,2,5\}$ does not comply with the case two.

(2)When the scheme is used in case three, the total public satisfaction is 52.214 , which is higher compared with other schemes. The time STD is 7.06, which is higher compared with other schemes. So the scheme $\{1,2,5\}$ can be suit for the case three but it is not the best scheme.

(2)According to the total satisfaction when two regions need emergency care, we can preliminary obtain the best ambulances configuration: $\{3,5,6\}$ and $\{4,5,6\}$.

(1)When consider scheme $\{4,5,6\}$, the total public satisfaction in case one and three are 3.925 and 39.117 respectively, which are lower compared to the other schemes. So the scheme $\{4,5,6\}$ does not meet the case one and three.

(2)When consider scheme $\{3,5,6\}$, the total public satisfaction in case one and three are 4.197 and 41.768 respectively, which are lower compared to the other schemes. So the scheme $\{3,5,6\}$ also does not meet the case one and three.

(3)According to the total satisfaction when three regions need emergency care, we can preliminary obtain the best ambulances configuration: $\{2,3,5\}$.

(1)When the scheme is used in case one, the total public satisfaction is 5.638 which is higher compared with other schemes. The time STD is 2.616, which is lower compared with other schemes. So the scheme $\{2,3,5\}$ is suit for the case one.

(2)When the scheme is used in case two, the total public satisfaction is 11.231 which is similar to other schemes. The time STD is 9.647, which is also similar to other schemes. So the scheme $\{2,3,5\}$ is suit for the case two.

In summary, the most reasonable solution is $\{2,3,5\}$, namely region $2,3,5$ place an emergency ambulance. The scheme is well in line with the above three conditions, with good public satisfaction, and also more reasonable at the time.

\section{References}

[1] Jia Yongxing, Research on the Problem of Dispatching Emergency Vehicles in the Disposal of Serious Traffic Accident Scene, Jilin University

[2] Wen Jiabao, Order of the State Council of the People's Republic of China No.495 < The Byelaw Governing Reporting, Investigation and Handling of Production Safety Accidents>

[3] Ji Yun, Logistic model and its application, Weifang higher occupation education 